\title{
Imaging ejecta from the final flash star V605 Aquilae
}

\author{
K. H. Hinkle ${ }^{1}$, T. Lebzelter ${ }^{2}$, R. R. Joyce ${ }^{1}$, S. Ridgway ${ }^{1}$, L. Close ${ }^{3}$, J. Hron ${ }^{2}$, and K. Andre ${ }^{2}$ \\ 1 National Optical Astronomy Observatories ${ }^{\star}$, PO Box 26732, Tucson, AZ 85726, USA \\ e-mail: [hinkle; joyce; ridgway] @noao.edu \\ 2 Institute for Astronomy, Türkenschanzstrasse 17, 1180 Vienna, Austria \\ e-mail: [lebzelter; hron; andre] @astro.univie.ac.at \\ 3 University of Arizona, Steward Observatory, Tucson, AZ 85721, USA \\ e-mail: 1close@as.arizona.edu
}

Received 27 April 2007 / Accepted 7 December 2007

\section{ABSTRACT}

\begin{abstract}
Aims. We investigated the cloud of ejecta resulting from the mass loss associated with the final helium shell flash in V605 Aql. Methods. V605 Aql was imaged at high spatial resolution in both optical emission lines and the infrared continuum using HST/WFPC2 and Gemini/Hokupa'a+QUIRC, respectively. The He I $10830 \AA$ A spectrum was also observed.

Results. The obscuring circumstellar shell, whose effects were first seen in 1923, is shown to be a disk with extended structure, including knots. The morphology of the V605 Aql circumstellar shell is discussed as an 80 year old in an evolutionary sequence consisting of recent, as well as much older, final flash objects.

Conclusions. The obscuration of V605 Aql by dust marked the emergence of the hot white dwarf remnant from an optically thick pseudo-photosphere. This white dwarf drives a $2500 \mathrm{~km} \mathrm{~s}^{-1}$ wind principally in one plane resulting in a circumstellar disk. Where the wind encounters the circumstellar disk, He I $10830 \AA$ emission is created, and hot, $\sim 1500 \mathrm{~K}$, grains are generated. Grains exit the disk at $350 \mathrm{~K}$, and the accompanying gas then expands at $\sim 140 \mathrm{~km} \mathrm{~s}^{-1}$. The strong concentration of the mass loss to the disk suggests the white dwarf is now rotating rapidly. There is convincing evidence in the literature that this process is also seen in V4334 Sgr and is still going on in the old final flash objects A30 and A78.
\end{abstract}

Key words. stars: evolution - stars: individual: V605 Aquilae - stars: mass-loss - stars: AGB and post-AGB stars: circumstellar matter - planetary nebulae: general

\section{Introduction}

Among the many successes of stellar evolution theory is the prediction of stars undergoing a "final flash". Final flash evolution occurs in stars that have terminated their AGB stage and are either on the constant luminosity post-AGB track or just entering the early white dwarf sequence. A final helium burning thermal pulse returns the star briefly to the AGB. The theory of these objects was initially developed early in the 1980s (Iben et al. 1983). In the late 1990s V4334 Sgr (Sakurai's star) was observed undergoing the predicted final flash behavior (Asplund et al. 1997). The final flash episode is astronomically very brief, hence the observation of a star undergoing a final flash is rare. However, the final flash stage is in fact a fairly common event in stellar evolution, occurring in $10 \%$ to $25 \%$ of all stars that evolve off the AGB (Blöcker 2001; Herwig 2005).

During the final helium shell flash stars revisit the AGB to post-AGB to white dwarf evolutionary sequence (see e.g. Herwig 2005; Werner \& Herwig 2006). In addition to the restoration of helium burning and the associated changes in luminosity, composition, etc., final flash stars have a renewed episode of mass loss. At the termination of the final flash the ejected material forms a planetary nebula about the stellar remnant. This second planetary nebula stage has been proposed to

\footnotetext{
* Operated by the Association of Universities for Research in Astronomy, under cooperative agreement with the National Science Foundation.
}

explain the shape and composition of some exotic multi-shell planetary nebulae (Jacoby \& Ford 1983; Iben et al. 1983).

As might be expected, very few stars are known that have clear evidence of current final flash evolution. Only three objects typically appear on the list: FGSge (Gonzalez et al. 1998), V605 Aql (Clayton \& de Marco 1997), and V4334 Sgr (Sakurai's object; Duerbeck \& Benetti 1996). Similar properties lead Clayton \& de Marco (1997) to suggest that final flash stars could be found in the enigmatic R CrB group. However, abundance results presented by Clayton et al. (2007) support a merged white dwarf origin for the $\mathrm{R} \mathrm{CrB}$ group. Among the three well documented final flash stars, V605 Aql and V4334Sgr have remarkable similarities (see Clayton et al. 2006, and references therein). The same brightening, spectral type evolution, and total obscuration by dust observed in V4334 Sgr during 1996-1999 was previously observed in V605 Aql during 1919-1923. V605 Aql is the central star of the old planetary nebula (PNe) A58. V4334 Sgr is also the central star of a very faint old planetary nebula.

The final flash can occur as a late thermal pulse (LTP) on the constant luminosity post-AGB track or as a very late thermal pulse (VLTP) on the early white-dwarf branch. In a VLTP the outer hydrogen layer is convected into the helium burning region and destroyed. This is not the case for a LTP (Werner \& Herwig 2006). The speed of the evolution of both V605 Aql and V4334 Sgr as well as the absence of hydrogen in their spectra argue for VLTP evolution. Models by Lawlor \& MacDonald (2003) link V4334 Sgr and V605 Aql with FG Sge as different 
evolutionary stages on a double loop VLTP path in the HR diagram. In these models a quick first return to the AGB is followed by evolution to the blue and a slow second return to the AGB. V4334 Sgr and V605 Aql would be on the first loop while FG Sge would be on the second, explaining the difference in evolutionary time scales. Lawlor \& MacDonald (2003) predicted that V605 Aql will cool back towards the AGB within the next 50-70 years and then resemble FG Sge. Similar two loop models are presented by Miller Bertolami et al. (2006). However, FGSge is more often identified as a LTP object as the surface abundances and time scale are not predicted correctly by a VLTP (Jeffrey \& Schönberner 2006).

V605 Aql was first detected in 1919 and initially classified as Nova Aquilae number 4 (Wolf 1920; Clayton et al. 2006). After brightening and fading from 1919 to 1923, 1924 saw V605 Aql dim beyond the range of the detectors then available (see Clayton et al. 2006, for a review). V605 Aql was largely forgotten until the 1980s. The one spectrum taken in 1921 shows V605 Aql to be a hydrogen-deficient carbon star (Clayton \& De Marco 1997). At the time of this report, more than 80 years after V605 Aql rose to brightness, $\mathrm{V} 605 \mathrm{Aql}$ is very faint visually at $V \sim 22$. In the infrared, however, V605 Aql is relatively brighter (Kimeswenger et al. 1998; Hinkle et al. 2001). Infrared measurements show that V605 Aql has become highly obscured in its own circumstellar cloud whose extinction $A_{\mathrm{v}}$ is at least 8 mag (Kimeswenger et al. 1998). The bolometric flux of V605 Aql remains similar to the value of the star discovered in 1919 (Kimeswenger et al. 2000). The time between the termination of the UV radiation exciting the $\mathrm{PNe}$, which corresponds to origin of the late He flash, and the rise to brightness in the 1920s must be astronomically brief (Lechner \& Kimeswenger 2004); the PNe is still observable.

In addition to shedding a dense cloud of dust, the final flash episode resulted in a drastic change in the surface composition of V605 Aql. Ingestion of surface hydrogen, predicted by VLTP theory and observed in V4334 Sgr, also occurred in V605 Aql. Spectroscopy of the V605 Aql PNe, A58, indicates a He/H ratio of $\sim 0.15$, while the circumstellar shell surrounding V605 Aql has a $\mathrm{He} / \mathrm{H}$ ratio of $\sim 1.2$ (Guerrero \& Manchado 1996).

In this paper we report on a multi-wavelength imaging study to explore the nature of the mass loss resulting from a final helium shell flash. V605 Aql was targeted because the roughly 8 decades since the initial event have allowed the ejecta to expand to a size where imaging is possible. Optical images have been reported by Guerrero \& Manchado (1996), Clayton \& De Marco (1997), and Bond \& Pollacco (2002). Clayton et al. (2006) suspected we might be viewing an optically thick torus of dust nearly edge on. Using the best available optical and infrared high-spatial resolution instrumentation for space and ground based imaging we report on a snapshot of the ejection of material.

\section{Observations}

\subsection{Images}

Three separate facilities (HST, Gemini North, and ESO) were used to obtain images of V605 Aql from visible through midinfrared wavelengths.

\subsubsection{HST/WFPC2}

V605 Aql was observed by HST/WFPC2 during a single orbit on 27 May 2001 UT. Total observation times were $1050 \mathrm{~s}$ and $460 \mathrm{~s}$ through the $502 \mathrm{~nm}$ and $658 \mathrm{~nm}$ narrow-band filters, respectively, broken into $350 \mathrm{~s}$ and $230 \mathrm{~s}$ individual exposures to aid in removal of cosmic ray artifacts. The filters were chosen to isolate the [O III] 5007 and [N II] 6584 lines.

The entire region of interest was contained on a single WF frame, so the additional reduction tasks consisted of combining the images at each wavelength using the IRAF "crrej" task to remove cosmic rays and rotation to position north and east in their nominal orientation. The HST images were then magnified to a pixel scale to match Hokupa'a/QUIRC infrared images and coaligned using two faint stars to the W of V605 Aql. They were then restored using the Lucy-Richardson algorithm, using the star approximately 10 arcsec SE of V605 Aql as the PSF standard. Twenty iterations were sufficient to decrease the FWHM of the PSF star from 0.23 arcsec to 0.086 arcsec.

\subsubsection{Gemini/Hokupa'a+QUIRC}

Infrared images were obtained at the 8-m Gemini North telescope with the University of Hawaii Hokupa'a adaptive optics system (Graves et al.1998, 2000) on the night of 20 June 2001 UT. Each observation consisted of multiple 2 min exposures with the QUIRC infrared imager, with telescope offsets of a few arcsec. The pixel scale for this instrument is 0.02 arcsec. A four position dither pattern with an integration time of $120 \mathrm{~s}$ was repeated twice for the $J$ and $K^{\prime}$ filters, four times in $H$. The total time on source was $960 \mathrm{~s}$ in $J, 1920 \mathrm{~s}$ in $H$, and $2160 \mathrm{~s}$ in $K^{\prime}$.

The multiple frames were median filtered to obtain the sky background. Standard IRAF imaging processing functions were used to sky-subtract, flat-field, shift, and coadd the images to produce a summed image. These images were then restored using the Lucy-Richardson algorithm within the STSDAS package. The resulting $F W H M$ of stars adjacent to V605 Aql was $0.17,0.14$, and 0.13 arcsec in the $J, H$, and $K^{\prime}$ filters, respectively.

The Gemini and HST images are shown in six panels of Fig. 1. The optical images $(1 \mathrm{~b}, 1 \mathrm{c})$ are in the forbidden lines [O III] 5007 and [N II] 6584. The [N II] filter used in WFPC2, $F 658 N$, is designed to exclude the $\mathrm{H} \alpha$ line. However, since $\mathrm{H} \alpha$ is a strong spectral line in the A58 PNe spectrum, the image of the PNe through the $F 658 N$ filter may include some $\mathrm{H} \alpha$ passed in the wings of the filter bandpass. Figure 2 shows the same data for the V605 Aql circumstellar nebula as contour plots.

\subsubsection{ESO/TIMMI2}

The mid-infrared multi-mode instrument TIMMI2 (Reimann et al.2000) on the 3.6-m telescope at ESO La Silla was used to image V605 Aql at $11.9 \mu \mathrm{m}$ with a pixel scale of $0.2^{\prime \prime}$. The observations were carried out on the night of 6 April 2001 UT. Nodding and chopping were done in perpendicular directions, producing 4 images of V605 Aql on the chip after the combination of all frames. The total exposure time per image was 1344 s. The data were reduced with the TIMMI2 pipeline (Relke et al.2000). The images of V605 Aql had a FWHM of $1^{\prime \prime}$ and were indistinguishable from images of point sources. Photometry was based on measurements of two standard stars. Since the $11.9 \mu \mathrm{m}$ image of $\mathrm{V} 605 \mathrm{Aql}$ is an unresolved point source, it is not illustrated. 


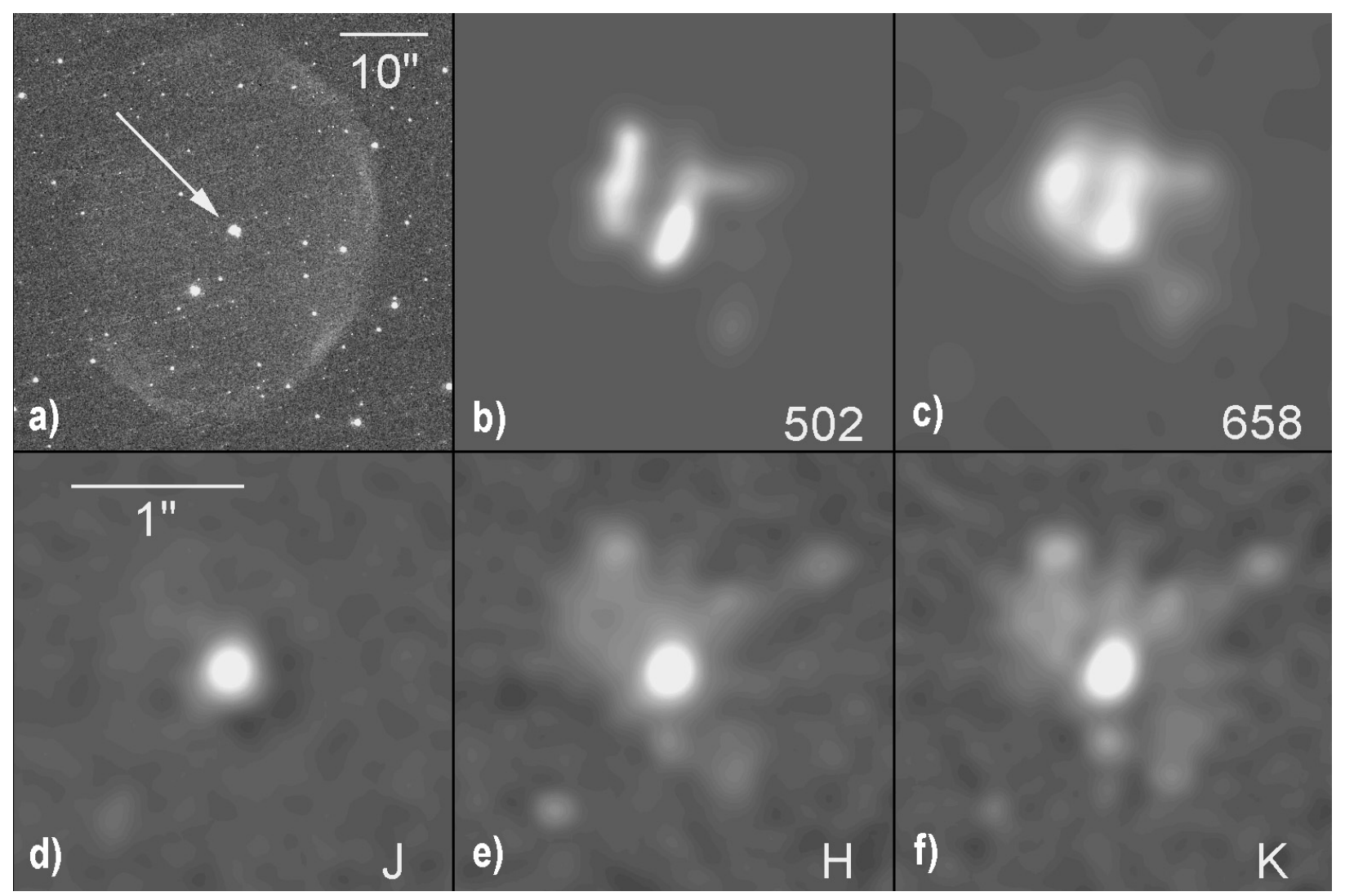

Fig. 1. a) Image of the A58 planetary nebula through the $F 658 N$ ([N II]) filter of WPFC2. V605 Aql is marked with an arrow. The image is 51 arcsec on each side. b), c) WFPC2 images of V605 Aql through the F502N ([O III]) and F658N ([N II]) filters, respectively. d), e), f) Hokupa'a/QUIRC images of V605 Aql through the $J, H$, and $K^{\prime}$ filters, respectively. The five closeup images are all 2.6 arcsec on a side. North is up and east to the left.
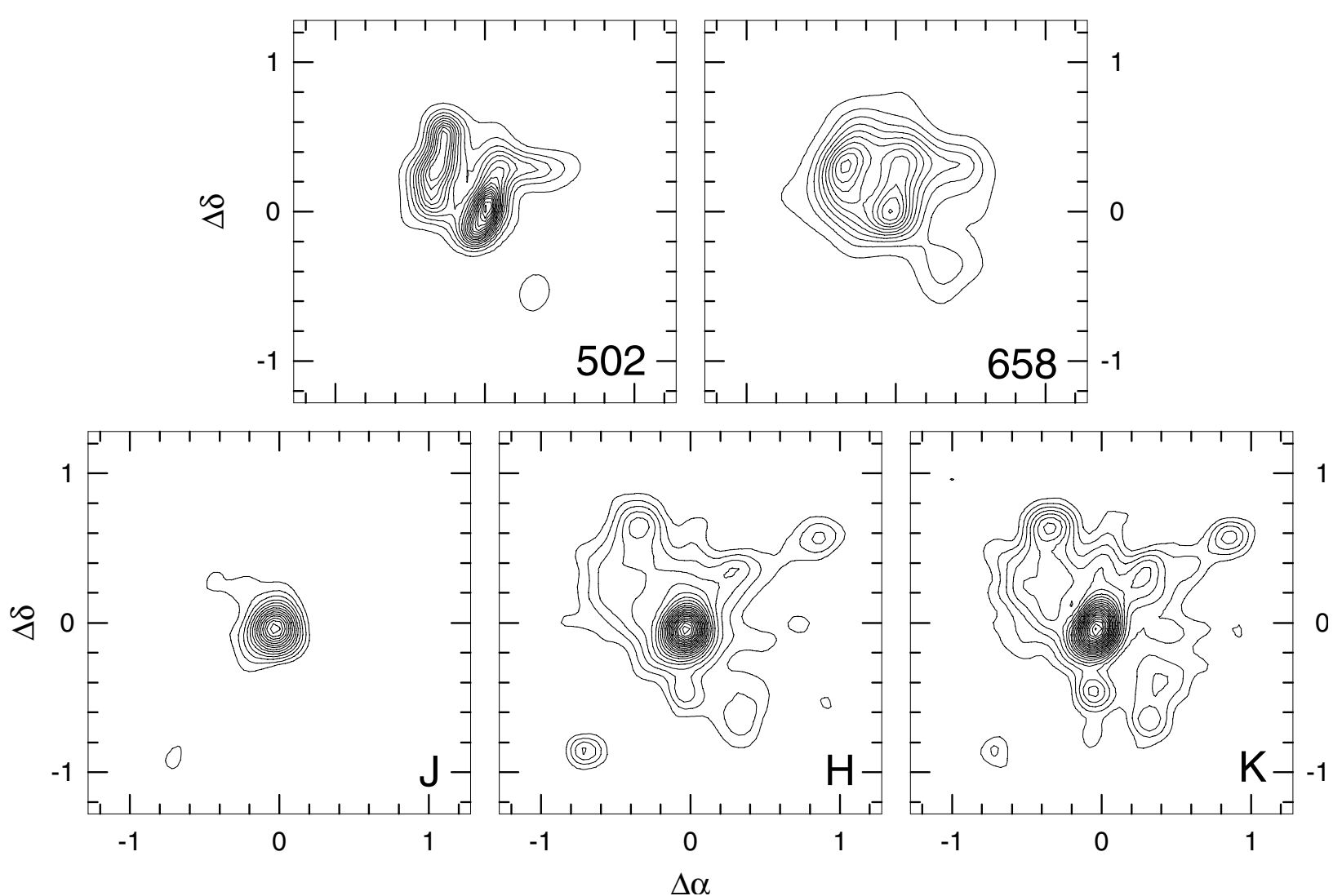

Fig. 2. Contour plots of V605 Aql in the F502N, F658N, J, H, and $K_{\mathrm{p}}$ bands. The images are 2.5 arcsec on a side to match the scale of Fig. 1. 


\subsection{Spectroscopy}

On 2000 Sep. 9 (UT) the medium-resolution cryogenic grating spectrograph CRSP (Joyce et al. 1994) was used on the KPNO $4 \mathrm{~m}$ telescope to obtain a $10 \mathrm{~min}$ observation of the 1.04 to $1.11 \mu \mathrm{m}$ region of the V605 Aql spectrum. The slit was opened to 2.2 arcsec to collect all of the flux from V605 Aql. The He I $10830 \AA$ line was observed in emission with peak $S / N=40$ at a seeing-limited spectral resolution $R \sim 670$. The A0V star HD 203856 was used for flux calibration.

\section{Images of V605 Aql}

Figure 1a shows the relation of the V605 Aql central nebula to the PNe A58. A58 has an age 23000 years (Guerrero \& Manchado 1996), a value in reasonable agreement with models of the time required to evolve from the tip of the AGB to the initiation of the final thermal pulse (Iben et al. 1983; Althaus et al.2005). A58 is slightly oblate. From ground-based images Lechner \& Kimeswenger (2004) estimate 50 arcsec for the A58 longitudinal axis and 38 arcsec for the A58 secondary axis. From the [NII] image in Fig. 1 we measure a major axis of 44 arcsec and a minor axis of 35 arcsec with a position angle of $\sim 166^{\circ}$. Pollacco et al. (1992) report this is a "middle-elliptical" morphology in Balick's (1989) classification scheme.

In both optical emission lines and near-infrared continuum (except perhaps $J$ ) the inner nebula of V605 Aql has a resolved circumstellar nebula with a maximum diameter of $\sim 1.6$ arcsec. The inner nebula as seen in the optical images has a clear orientation with dark band of the central knot at a major axis position angle of $\sim 150^{\circ}$ (Fig. 1b,c). In all the images an unresolved central point source dominates the flux.

The distance of V605 Aql can be estimated from the A58 planetary nebula using assumptions for typical PNe. There is considerable uncertainty in this method when applied to the distance of an individual object. Distances for A58 found in the literature range upwards from $2.7 \mathrm{kpc}$ (Cahn \& Kaler 1971) with values clustering around 3.5 to $4 \mathrm{kpc}$. In general agreement, Lechner \& Kimeswenger (2004) find a distance of $3.1 \mathrm{kpc}$ from radio observations. A limiting distance of $5.1 \mathrm{kpc}$ is derived from the infrared flux measured by IRAS (van der Veen et al. 1989). Koller \& Kimeswenger (2001) note that the stellar luminosity is in much better agreement with evolutionary models if the distance is near the $5 \mathrm{kpc}$ limit. In the following discussion we will consider both a standard distance of $3.8 \mathrm{kpc}$, from a mass-radius relation and radio measurements at $5 \mathrm{GHz}$ (Maciel 1984), and a $5 \mathrm{kpc}$ distance.

\subsection{Optical forbidden line images}

The [O III] and [N II] HST images reveal an extended, clumped nebula and an unresolved central point source. There are two previously reported images of the central nebula of V605 Aql. In 1991 before the HST repair mission, the HST Faint Object Camera was used to observe V605 Aql in [O III] (Clayton \& De Marco 1997; Bond \& Pollacco 2002). The raw image has very similar morphology to the image obtained in 2001 . However, the diameter of the nebula is only 0.6 arcsec (Bond \& Pollacco 2002), 2.7 times smaller than the diameter in 2001. At a distance of $3.8 \mathrm{kpc}$, the implied expansion velocity is $1800 \mathrm{~km} \mathrm{~s}^{-1}$. Unfortunately the quality of the 1991 image is poor.

A ground based optical image in the [N II] $6584 \AA$ line was obtained under conditions of excellent seeing
(Guerrero \& Manchado 1996). The resolution of this image, about 0.6 arcsec, is considerably less than that of the current data, but it does reveal structure after subtraction of the point spread function. The alignment of this structure and the reported diameter (1.4 arcsec) agrees with the current result (the Guerrero \& Manchado 1996 PA is aligned through the two bright knots while our PA is rotated approximately $90^{\circ}$ and aligned along the dark lane). As noted by Guerrero \& Manchado (1996), the axial symmetry displayed in V605 Aql is strongly suggestive of a bipolar structure.

\subsection{Optical spectroscopy}

The optical spectrum of V605 Aql consists entirely of emission lines. Three widths of lines are present, broad lines with $F W H M \sim 2600 \mathrm{~km} \mathrm{~s}^{-1}$, nebular lines of $F W H M \sim$ $200 \mathrm{~km} \mathrm{~s}^{-1}$, and narrow PNe lines (Pollacco et al. 1992; Guerrero \& Manchado 1996; Kimeswenger 2003; Clayton et al.2006). The broad C IV $5806 \AA$ line defines the [WR] spectral type of the obscured stellar remnant ${ }^{1}$. Optical spectra obtained in 2001 show in addition three broad C IV lines, two He II lines and one O V line from the stellar remnant (Clayton et al. 2006). Line ratios set $T_{\text {eff }} \sim 95000 \mathrm{~K}$. The [WR] line widths imply a wind velocity of $2500 \mathrm{~km} \mathrm{~s}^{-1}$ and a central source mass loss rate of $1.3 \times 10^{-8} M_{\odot} \mathrm{yr}^{-1}$. There is no evidence for hydrogen in the atmosphere of the [WR] star. Kimeswenger (2003) notes that these broad lines are centered at the systemic velocity of the PNe A 58. This implies that the lines are scattered by a symmetric expanding dust shell. Clayton et al. (2006) note that the [WR] spectrum must be scattered by the nebula since it is not possible to image the [WR] star.

The optical images in Fig. 1 are in nebular [O III] and [N II] lines. Pollacco et al. (1992) report on moderate resolution $(R \sim 14000)$ spectroscopy of the central knot. The [O III] and [N II] lines have $F W H M \sim 270$ and $180 \mathrm{~km} \mathrm{~s}^{-1}$ respectively. The PNe lines, which are also present in the spectrum, have unresolved widths but are doubled showing an expansion velocity of $31 \mathrm{~km} \mathrm{~s}^{-1}$ (Pollacco et al. 1992). Using the systemic velocity derived from the PNe spectrum, the central knot emission lines have an expansion velocity of $\sim 140 \mathrm{~km} \mathrm{~s}^{-1}$ (Guerrero \& Manchado 1996). These lines have only one blue shifted velocity component, i.e. they originate in the side of the nebula toward us. The back side of the nebula is obscured (Pollacco et al. 1992). Lower resolution spectroscopy by Guerrero \& Manchado (1996) confirm the $\sim 200 \mathrm{~km} \mathrm{~s}^{-1}$ expansion velocity. The nebula has an electron density of $\sim 2600 \mathrm{~cm}^{-3}$ with an electron temperature of $12500 \mathrm{~K}$ (Guerrero \& Manchado 1996).

The excitation mechanism for the $\sim 200 \mathrm{~km} \mathrm{~s}^{-1}$ wide optical lines has been discussed by Guerrero \& Manchado (1996) for V605 Aql and more recently by Kerber et al. (2002) for similar lines observed in the spectrum of V4334 Sgr. Based on line ratios compared to models presented by Hartigan et al. (1987), Guerrero \& Manchado (1996) concluded that the V605 Aql lines were shock excited. This is very appealing because the line widths in the Hartigan et al. (1987) models nicely match the observed line widths in V605 Aql. However, both the line ratios to $\mathrm{H} \alpha$ and the comparison to solar abundance models presented by Guerrero \& Manchado (1996) seem questionable since V605 Aql is now known to be very deficient in hydrogen. Kerber et al. (2002) found that in the case of V4334 Sgr the evidence

\footnotetext{
1 The [WR] classification system of van der Hucht et al. (1981) is used for the emission-line low-mass central stars of PNe.
} 


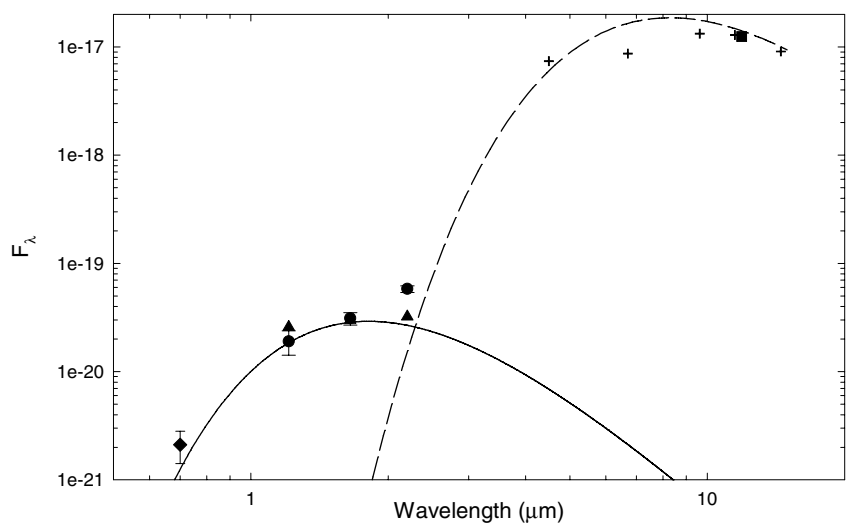

Fig. 3. Spectral energy distribution of V605 Aql over the range 0.6-15 $\mu \mathrm{m}$. Filled diamond: Seitter (1987); filled circle: Hinkle et al. (2001); filled triangle: this paper Hokupa'a+QUIRC; plus: Kimeswenger et al. (1998); filled box: this paper TIMMI2. The solid and dashed lines are illustrative blackbody fits at $1500 \mathrm{~K}$ and $350 \mathrm{~K}$, respectively (see text). The $0.7 \mu \mathrm{m}$ point has not been used in the fit. The $J$ band fluxes may be contaminated by He I $10830 \AA$ emission (see text).

was not sufficient to decide between photoionization and shock excitation.

\subsection{Infrared images}

The $K$ and $H$ band near infrared images, like the visual images, show an extended circumstellar shell around V605 Aql. The $J$ band image shows only a slight hint of extension, and is almost a point source. This image certainly marks the position of V605 Aql, and using two faint stars to the west as fiducials, we confirm that this location is the brightest object in all of the other images.

Infrared photometry derived from the central point source in the images is shown in Fig. 3, along with some previous photometry for V605 Aql. The $11.9 \mu \mathrm{m}$ measurement of the central point source is in nearly perfect agreement with photometry from the 20 arcsec beam of ISO (Kimeswenger et al. 1998), demonstrating that all the mid-IR flux comes from the central point source. The lack of spatial structure of the $11 \mu \mathrm{m}$ images should not be taken as an indication that extended structure does not exist. Rather, it probably indicates that the current sensitivity of $11 \mu \mathrm{m}$ imaging is sufficient to detect the bright central point source but not the much fainter diffuse radiation from the surrounding nebula.

In the near infrared, comparison of the current results to previous larger beam results shows that the central point source also dominates the flux. Assuming that the photometry, past and present, is dominated by the central point source, a simple blackbody fit to the energy distribution requires at minimum a two temperature model; in Fig. 3 we fit 350 and $1500 \mathrm{~K}$ blackbodies to the photometry. Whitelock (1985) finds that $J$ band fluxes of PNe can have up to $50 \%$ contributions from He I $10830 \AA$. As shown below, He I $10830 \AA$ is a very strong feature in the spectrum of V605 Aql. However, we find that the He I flux accounts for only a few percent of the $J$ band signal in the Hinkle et al. (2001) V605 Aql photometry.

Blackbody fits to the photometric data yield only very approximate dust temperatures. Both improved photometry as well as detailed modeling including grain properties are required to extract detailed information. Koller \& Kimeswenger (2001) noted that a single temperature, single grain size model does not fit the IRAS/ISO photometry for V605 Aql but that a good fit over the 3.5 to $100 \mu \mathrm{m}$ wavelength range can be made by using a distribution of grain sizes heated by the central star. The related object $\mathrm{A} 30$ has a similar infrared spectrum. Borkowski et al. (1994) provide a similar discussion of grain sizes and heating of the grains by the central star.

\section{Comparison with other final flash objects}

Observations of V605 Aql and V4334 Sgr were first recorded at roughly the same stage of the final flash process. While the final flash evolution of these stars is remarkably similar, FG Sge is undergoing much slower final flash evolution. The SED of FG Sge is quite different from that of either V605 Aql or V4334 Sgr with a circumstellar shell temperature of $\sim 1000 \mathrm{~K}$ (Gehrz et al. 2005). As noted in Sect. 1, FG Sge has been discussed as being either in LTP evolution (Werner \& Herwig 2006) or on the second loop of VLTP evolution (Lawlor \& MacDonald 2003).

In addition to these three objects, four other objects are known which have hydrogen-rich outer PNe and hydrogen-poor inner regions. In these objects the central stars are type [WC]. CK Vul was suggested by Evans et al. (2002) to be a 300 year old final flash object. However, further observations are required to fully explore the nature of this object (van Hoof et al. 2006). On the other hand, A30 and A78 are well documented with kinematic ages for the hydrogen poor knot of at least $10^{3}$ years (Harrington et al. 1995). Guerrero \& Manchado (1996) suggest an age nearer $10^{4}$ years for the hydrogen poor inner knot around A78. IRAS 15154-5258 is also in this class of objects with a kinematic age of less than $10^{3}$ years (Zijlstra 2002).

Models by Blöcker \& Schönberner (1997) give massdependent lifetimes of $>10^{3}$ years for thermal pulse evolution counting from departure from the white dwarf track to the return. Using departure from the white dwarf track to define thermal pulse evolution, all seven objects listed above would then be in different phases of late-thermal pulse evolution. Faster thermal pulse evolution, as reviewed by van Hoof et al. (2007) implies a return to the white-dwarf track in a few hundred years. In any case, the seven objects make up an observation related set bearing in mind evolutionary dependence on mass and uncertainties concerning VLTP versus LTP evolution.

From, for instance, the number of observed PNe in the solar neighborhood and the above models, the sample of known final flash stars would seem highly incomplete. The existence of a much larger sample of final flash stars seems supported by the relatively recent detections of V605 Aql and V4334 Sgr undergoing a final flash. These detections suggest that stars undergoing rapid variations during the final flash are much more readily detected than other final flash objects. Helium-rich central stars with hydrogen-rich PNe form a much larger sample (e.g. Górny 2001). The deficit of helium-rich inner PNe possibly results from the rapid dissipation of the helium-rich ejecta into the much greater mass of the hydrogen-rich ejecta. For instance, van Hoof et al. (2007) suggests that the mass ejected by V4334 Sgr in the VLTP eruption is between $10^{-4}$ and $10^{-2} M_{\odot}$. The hydrogen-rich ejecta originate during the AGB mass-loss stage where $\sim 1 M_{\odot}$ or more is ejected (Herwig et al. 1999). However, helium-rich central stars also can result from alternative evolutionary routes (Górny \& Tylenda 2000).

Six years after the first observation of the final flash, V4334 Sgr was fully obscured in an optically thick, circumstellar dust shell of 40-50 mas diameter with a (gas) expansion 
Table 1. Observations of final flash stars.

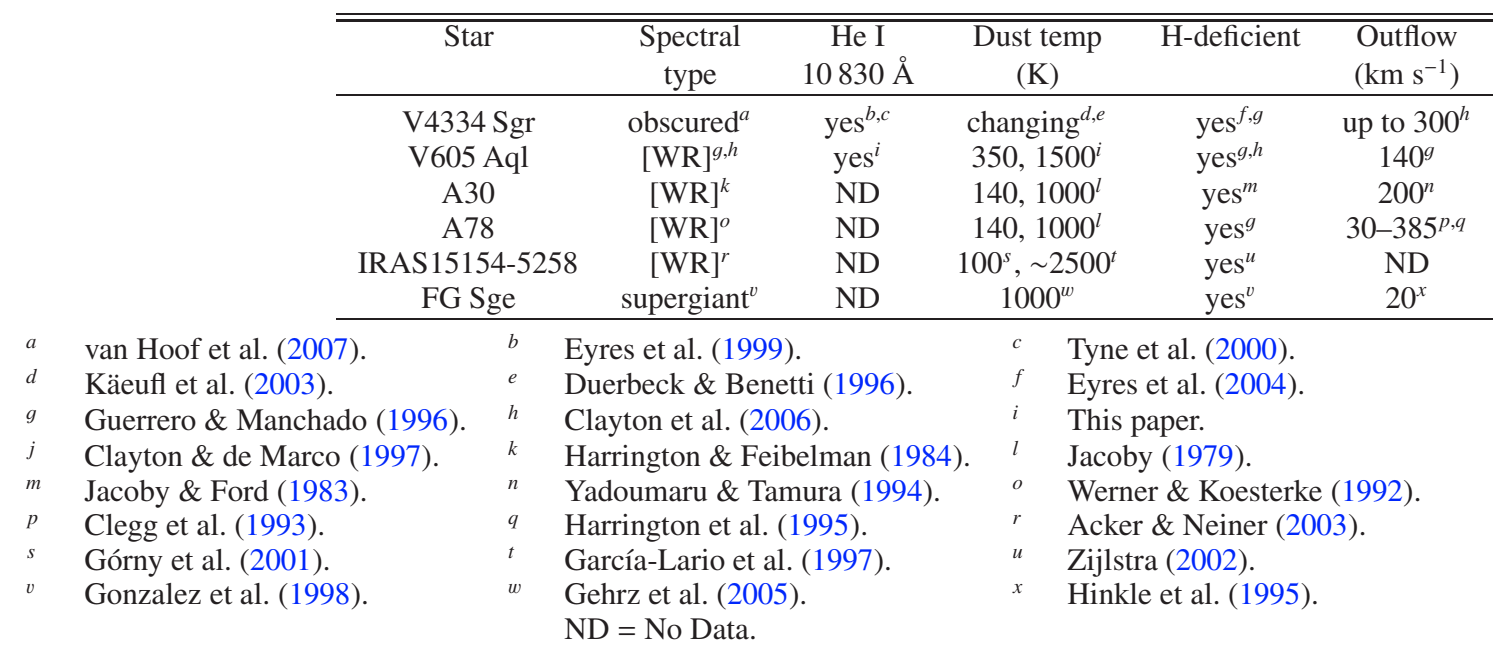

velocity $49 \pm 5 \mathrm{~km} \mathrm{~s}^{-1}$ (Tyne et al. 2002). The production of copious amounts of dust around V4334 Sgr was monitored by midIR photometry (Käufl et al. 2003). Clayton et al. (2006) note that when dust forms around final flash stars the abundances, temperature, absolute luminosity, and light curve behavior are nearly indistinguishable from an RCB star. We will refer to this dust forming stage in final flash stars as the RCB stage.

As discussed above, V605 Aql underwent an RCB stage in the 1920s and has been dust enshrouded since that time. Millennia after their final flash, IRAS 15154-5258, A30, and A78 remain dust enshrouded with a complex hydrogen-deficient nebula interior to a nearly spherical outer planetary. For both A30 and A78 the inner nebula is $50-60 \%$ of the diameter of the outer nebula. In the case of the younger IRAS 15154-5258 the $\mathrm{H}$-poor inner nebula is $\sim 25 \%$ the diameter of the H-rich nebula (Zijlstra 2002).

The interior of the A30, A78, and IRAS 15154-5258 nebulae have knots and cometary structures from interaction with the wind from the hot central star (Borkowski et al. 1993). Both A30 and A78 have multiple velocities present. The outer PNe of A78 is expanding at $40 \mathrm{~km} \mathrm{~s}^{-1}$. A central disk like structure has knots expanding at $30 \mathrm{~km} \mathrm{~s}^{-1}$ (Clegg et al. 1993). A pair of polar knots are moving perpendicular to the ring at $\pm 100 \mathrm{~km} \mathrm{~s}^{-1}$ and polar ejecta is expanding at velocities up to $\sim 385 \mathrm{~km} \mathrm{~s}^{-1}$ (Harrington et al. 1995). The [WR] central star of A78 has a stellar wind with terminal velocity $3600 \mathrm{~km} \mathrm{~s}^{-1}$ and mass loss rate $2.5 \times 10^{-8} M_{\odot} \mathrm{yr}^{-1}$ (Werner \& Koesterke 1992).

Near-IR images of A30 show hot dust in an expanding equatorial ring of H-poor gas (Dinerstein \& Lester 1984; Borkowski et al. 1994). Three sets of velocities are present in A30, a $40 \mathrm{~km} \mathrm{~s}^{-1}$ outflow for the PNe, a $>200 \mathrm{~km} \mathrm{~s}^{-1}$ outflow in the central region (Yadoumaru \& Tamura 1994) and a $4000 \mathrm{~km} \mathrm{~s}^{-1}$ stellar wind. The mass loss rate for the A30 is $5 \times 10^{-8} M_{\odot} \mathrm{yr}^{-1}$ (Leuenhagen et al. 1993). The complex structure seen in both A30 and A78 is attributed to the fast stellar wind from the central [WR] star blowing a bubble in the surrounding dust shell (Meaburn et al. 1998). The clumps in the bubble are RayleighTaylor unstable and in the [WR] wind clump fragmentation occurs with the clumps eventually accelerated by the stellar wind (Borkowski et al. 1995).

We present a summary of the properties of the final flash objects in Table 1.

\section{Discussion}

The [WR] spectrum of V605 Aql originates in a wind off the central star with a velocity of several thousand $\mathrm{km} \mathrm{s}^{-1}$ (Clayton et al. 2006). The hydrogen-deficient circumstellar shell has an expansion velocity of $\sim 140 \mathrm{~km} \mathrm{~s}^{-1}$ (Pollacco et al. 1992). The hydrogen-rich PNe has an expansion velocity of $31 \mathrm{~km} \mathrm{~s}^{-1}$ (Pollacco et al. 1992). These multiple velocities suggest that an interacting wind model (Kwok 1982) applies to the V605 Aql circumstellar shell. In its simplest form the interacting wind model is a bubble of circumstellar material surrounding the central star, as described above for A30 (see also Pollacco et al. 1992). However, the observation of the [WR] lines proves that holes exist and combined with the bi-polar morphology suggest a torus-like structure (Pollacco et al. 1992; Clayton et al. 2006). The more evolved objects A30 and A78 also suggest an equatorial ring and polar knots (Borkowski et al. 1995, Harrington et al. 1995). We will show below that V605 Aql is indeed surrounded by a circumstellar disk and that this same model in all likelihood applies to all the known final flash objects with the possible exception of FG Sge.

\subsection{He / $10830 \AA$}

An important clue to understanding V605 Aql are observations of He I $10830 \AA$. He I $10830 \AA$ is a triplet transition to a metastable lower level $20 \mathrm{eV}$ above the ground state. LTE population of this transition requires temperatures $\sim 20000 \mathrm{~K}$. The He I line in V4334 Sgr appeared at the time of the first dust formation in the atmosphere (Tyne et al.2002) and indicates a wind velocity of $\sim 670 \mathrm{~km} \mathrm{~s}^{-1}$ (Eyres et al. 1999). This wind has been observed only in He I. Similar He I $10830 \AA$ profiles can be found in some RCB stars. The He I $10830 \AA$ line in RCB stars is believed to be formed in shocks as dust clouds are radiatively driven from the star (Clayton et al. 2003).

V605 Aql in the early 1920's underwent dimming episodes associated with dust cloud formation in RCB objects (Clayton et al. 2006). Figure 1e,f shows several knots surrounding the central infrared source. If we assume these clouds condensed in 1922 and are moving tangentially, the radial distances in the images and a distance of $3.8 \mathrm{kpc}$ require cloud velocities of $\sim 180 \mathrm{~km} \mathrm{~s}^{-1}$. So the dust knots in the images could be ejected dust clouds from the RCB stage of V605 Aql. 


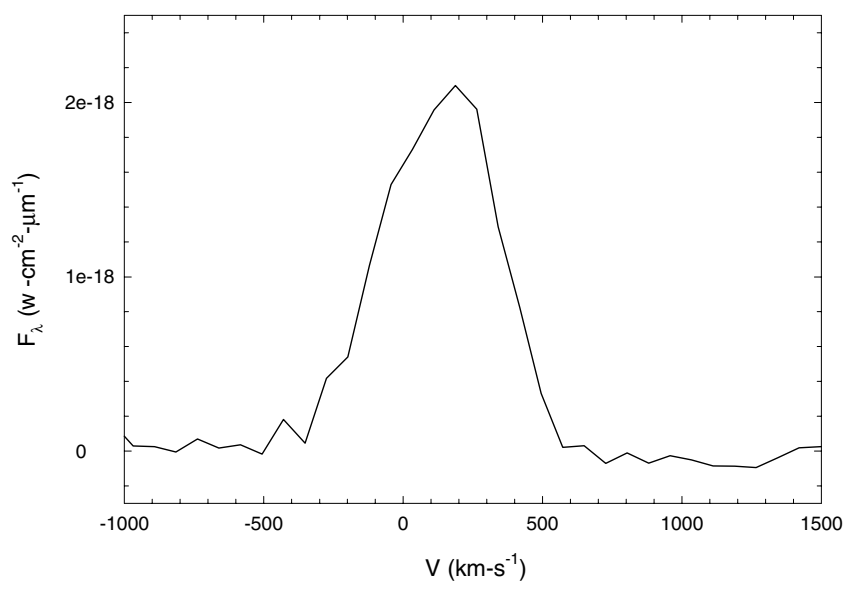

Fig. 4. He I 10830 Å observed in V605 Aql on 9 Sep. 2000. The velocity scale is heliocentric. The line has a $F W H M$ of $\sim 500 \mathrm{~km} \mathrm{~s}^{-1}$.

However, He I $10830 \AA$ has been reported in the spectrum of V4334 Sgr after V4334 Sgr was totally obscured (Hinkle \& Joyce 2002; Tyne et al. 2000). In Fig. 4 we show the He I 10830 A line in V605 Aql 80 years after this final flash star was obscured. Since V605 Aql and V4334Sgr are no longer shedding dust clouds, we propose that the shock generating the He line occurs as the [WR] wind encounters the dust shell and dust grains condense. In both V4334 Sgr and V605 Aql the He I $10830 \AA$ line has a full width at zero intensity of $\sim 600 \mathrm{~km} \mathrm{~s}^{-1}$. Large shifts of the line profile from the outflow velocity are recorded for V4334 Sgr, in keeping with a shock termination of the [WR] wind where the shock zone is on a line of sight to the observer. In V605 Aql the He I line center is shifted $\sim+130 \mathrm{~km} \mathrm{~s}^{-1}$ from the systemic velocity requiring that it be formed on the far side of the star from the observer.

\subsection{The origin of the infrared radiation}

The behavior of dust from an episodic ejection is well known from the study of classical novae (Gehrz 1999). Classical nova undergo nuclear burning increasing the luminosity which approaches or exceeds the Eddington limit. van Hoof et al. (2007) point out that the linear momentum in the ejecta from V4334 Sgr exceeds that in the stellar radiation so the star is susceptible to catastrophic mass loss. In nova this results in the ejection of a expanding shell of material. In the case of an extreme $\mathrm{CO}$ nova dust condenses in the ejecta and forms an optically thick dust shell. The dust shell cools and disperses after a period of months (see Gehrz 1999).

An amazing feature of Fig. 1 is that we can see near-infrared radiation from dust in V650 Aql 80 years after the dust forming event took place. Gehrz et al. (2005) similarly noted the same unexpected near-infrared brightness in FG Sge. In FG Sge twenty years after the dust formation event the dust remains hot, near the condensation temperature for grains. Gehrz et al. (2005) proposed that the dust formation event in FGSge was not a shell ejection but rather the establishment of an optically thick wind. In FG Sge the grains form at the $1000 \mathrm{~K}$ condensation zone at the base of the outflow.

Figure 3 demonstrates that the dust in V605 Aql can be approximately fit by two temperatures, $\sim 1500 \mathrm{~K}$ and $\sim 350 \mathrm{~K}$. The $1500 \mathrm{~K}$ component is at the condensation temperature of graphite dust with $\mathrm{C} / \mathrm{O} \sim 1.05$; larger $\mathrm{C} / \mathrm{O}$ equates to higher condensation temperatures (Sharp \& Wasserburg 1995). Dust

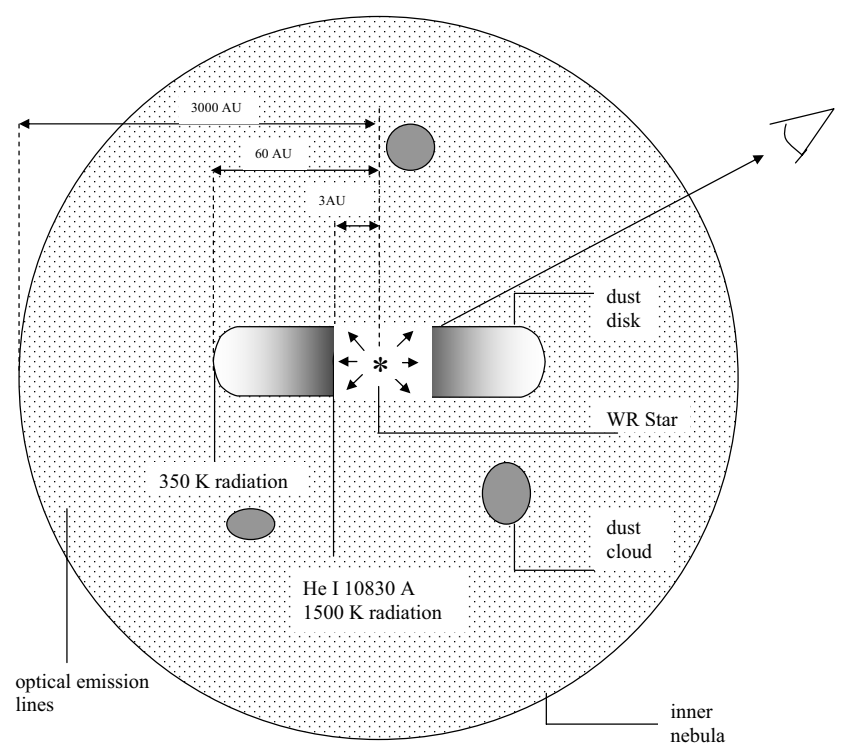

Fig. 5. A schematic illustration of the V605 Aql circumstellar shell. The $3.8 \mathrm{kpc}$ distance is assumed. Components are not shown to scale. The disk is seen inclined to the line of sight. The geometry of the inner disk dust wall is restricted to nearly perpendicular to the plane of the disk since the opposite side of the inner dust wall is directly observed but the central star is not directly observed.

is known to form in the wind of typical [WR] stars (Górny et al.2001). We propose that in the case of V605 Aql, as in FG Sge, dust is being continuously condensed out of the stellar wind and that $1500 \mathrm{~K}$ marks the condensation radius of the dust.

As discussed in Sect. 5.1, we propose that the condensation point in V605 Aql results in the shock marked by He I $10830 \AA$ emission. We can see the shock zone because the V605 Aql dust shell has the form of a disk inclined to the line of sight (Guerrero \& Manchado 1996). In order to allow the back side of the inner disk to be seen but not the central star the disk must be thick relative to the diameter of the central star. Also, the walls of the disk must be roughly perpendicular to the plane of the disk (see for example Dominik et al. 2003). We propose $\sim 350 \mathrm{~K}$ is the temperature where the dust shell becomes optically thin at midinfrared wavelengths.

For FG Sge Gehrz et al. (2005) extracts the physical properties of the dust shell from the photometry. By assuming a spherical circumstellar shell the inner and outer diameters of the disk can be computed from the stellar luminosity and dust temperature. Following Clayton et al. (2006) we assume a stellar luminosity of $10^{4} L_{\odot}$. In this simple model the dust condenses at approximately $5 \times 10^{13} \mathrm{~cm}(3 \mathrm{AU})$ and becomes optically thin at $9 \times 10^{14} \mathrm{~cm}(60 \mathrm{AU})$. A sketch of the V605 Aql circumstellar region based on these numbers is shown in Fig. 5 .

Hot dust is a feature of $\sim 6 \%$ of PNe (Phillips \& RamosLarios 2006). Dust in PNe and the dust in the extended V605 Aql clouds must be heated directly; the infrared radiation can not be reprocessed radiation. For instance, if we consider the observed $K$ band flux in V605 Aql as reprocessed energy radiating at the $1500 \mathrm{~K}$ (Fig. 3), the spectral radiance at $2.2 \mu \mathrm{m}$ of $3.9 \mathrm{~W} \mathrm{~cm}^{-2} \mu \mathrm{m}^{-1} \mathrm{sr}^{-1}$, coupled with the observed $K$ magnitude $\left(K=14.6\right.$, i.e. $\left.5.6 \times 10^{-20} \mathrm{~W} \mathrm{~cm}^{-2} \mu \mathrm{m}^{-1}\right)$ yields a radius of the emitting region of $1.4 \times 10^{-5}$ arcsec. Material in grey body equilibrium at an apparent distance of $0.1 \operatorname{arcsec}$ will then be at a temperature of $\sim 19 \mathrm{~K}$. In such a case, the contribution of the 
thermal flux to the $2 \mu \mathrm{m}$ image would be negligible. Phillips \& Ramos-Larios (2006) suggest that photon absorption by very small grains is an effective heating mechanism. Beyond the requirement for suitably sized grains, grain destruction by UV is a problem with this model. Clearly modeling with a range of grain sizes and including absorption and re-emission by dust is required (see for example Misselt et al. 2001).

It is unlikely that any of the infrared flux comes from light from the central star escaping along the poles of the circumstellar disk. Light escaping at the poles would be scattered by the surrounding dust and produce an infrared reflection nebula such as those seen in protostellar objects. Polarimetric imaging would be a diagnostic of this contribution to the flux, since reflection nebulosities are highly polarized (Clayton et al.2006). The V605 Aql nebula is unlike most reflection nebulae, as it is significantly redder than the central point source (and even more so than the central star) and is relatively uniform in color. This would require an unlikely extinction geometry. The reddening of the extended regions is obvious in the $J$ band image (Fig. 2), which is notable for its lack of spatial extent.

Other than the HeI $10830 \AA$ region reported on here (Fig. 4) there are no existing near-infrared spectra of V605 Aql. However, the spectrum of V4334 Sgr in the $1-2.5 \mu \mathrm{m}$ region is featureless except for He I $10830 \AA$ (Hinkle \& Joyce 2002). It is unlikely that there would be infrared fluorescence lines in the near-IR of these objects. The inner nebulae are very hydrogen poor (Clayton et al. 2006) so the principal molecule producing fluorescence, $\mathrm{H}_{2}$, is extremely depleted. In RCB stars the hydrogen-deficient environment excludes the formation of PAHs and results in a nearly featureless 3-25 $\mu \mathrm{m}$ spectrum (Lambert et al. 2001).

We suggest that the outlying infrared sources in Fig. 1 are clouds ejected in the RCB stage of V605 Aql. Heating of these clouds poses a much more difficult challenge to understanding V605 Aql than the heating of the central source. Given the number of velocities present in the V605 Aql nebula as well as the range of velocities measured for dust clouds in more evolved final flash objects, for example A78 (Sect. 4), it seems very likely that the dust clouds encounter winds of very different velocities and shocks develop. It seems likely that the observed hot dust is the result of these shocks. Draine (1981) found that grains will not be significantly heated by a shock around a [WR] star but grain heating by collision with the shock excited gas ions has been shown to be an effective heating agent for the grains in A30 and A78 (Borkowski et al. 1993). The existence of hot dust in many PNe (Phillips \& Ramos-Larios 2006) suggests that this is an important and ubiquitous process.

\subsection{Mass loss}

In the preceding section we have seen that mass loss in V605 Aql is not an instantaneous event but a prolonged outflow. Measuring the mass loss of V605 Aql is of interest since the mass ejected directly influences the nature of the remnant. Models by Miller Bertolami et al. (2006) suggest the ejected material includes the convective envelope. Values for the envelope mass are $\sim 10^{-3} M_{\odot}$ (Iben 1984). The outer convective layers were found to have a mass of a few times $10^{-4} M_{\odot}$ (Miller Bertolami et al. 2006).

Clayton et al. (2006) find a mass loss rate of $1.3 \times$ $10^{-7} M_{\odot} \mathrm{yr}^{-1}$ for the [WR] star. For the [WR] star at the center of A78 Kaler et al. (1988) find $\dot{M} \sim 10^{-8} M_{\odot} \mathrm{yr}^{-1}$. Górny et al. (2001) finds a typical [WR] mass loss rate of $10^{-6} M_{\odot} \mathrm{yr}^{-1}$ but Górny \& Tylenda (2000) note that the final-flash [WR] stars are probably not typical of the [WR] stars at the center of PNe. If, as suggested above, the V605 Aql [WR] wind has been constant for the last 80 years then the Clayton et al. (2006) value of $1.3 \times 10^{-7} M_{\odot} \mathrm{yr}^{-1}$ applies over this period and the wind has removed $\sim 1 \times 10^{-5} M_{\odot}$ thus far from the star.

Observations in the infrared for V4334 Sgr allowed the first detection of $\mathrm{CO}$. Assuming mass loss driven by radiation pressure on dust, the gas is dragged along in the mass flow. The $\mathrm{CO}$ velocities then measure the bulk flow just ahead of the optically thick dust continuum (Eyres et al. 2004). In the four years after the dust was first observed the $\mathrm{CO}$ was observed to accelerate from $\sim 10 \mathrm{~km} \mathrm{~s}^{-1}$ (Pavlenko et al. 2004) to $\sim 300 \mathrm{~km} \mathrm{~s}^{-1}$ (Eyres et al.2004). Absorption CO lines were observed, hence the velocity is heavily weighted to the line of sight radial velocity. The mass loss rate for V4334 Sgr can be estimated from the CO observations of Eyres et al. (2004) by using the CO column density $\left(7 \times 10^{17} \mathrm{~cm}^{-2}\right)$ and velocity $\left(290 \mathrm{~km} \mathrm{~s}^{-1}\right)$. Taking the Asplund et al. (1999) $\mathrm{C} / \mathrm{He}$ ratio of 50, a stellar radius appropriate for $0.6 M_{\odot} \mathrm{G}$ supergiant, and a scale height appropriate for this atmosphere (Kurucz 1979), the mass loss rate is $\sim 10^{-7} M_{\odot} \mathrm{yr}^{-1}$. This is in excellent agreement with the [WR] stage values from Clayton et al. (2006) for V605 Aql.

\subsection{Optical images}

Morphological differences between the infrared and optical images of the V605 Aql are a conspicuous feature of Fig. 1. In particular the central region has a dark lane in the optical and a bright point source in the infrared. Comparing the images of V605 Aql against, for instance, those of known bipolar sources with dust disks, (e.g. HD 44179 - Cohen et al. 2004) the optical dark lane in V605 Aql has the appearance of the shadow of the dusty disk. The dusty disk is the main source of the infrared radiation. As noted by Clayton et al. (2006), the central [WR] star is obscured by the disk and is not visible.

The V605 Aql nebular emission lines have peak velocities, which sample a broad range of angle from the center, of $\sim 140 \mathrm{~km} \mathrm{~s}^{-1}$ (Guerrero \& Manchado 1996). The gas seems well distributed in the image and not a shell from a single ejection event. This suggests that the nebular gas has been ejected in the ongoing stellar wind. The extent of the gas also demands that we are not seeing a collimated bipolar flow from the side. If we assume that the actual velocity of the gas is near $140 \mathrm{~km} \mathrm{~s}^{-1}$, this matches the He I $10830 \AA$ velocity, and the gas is the post-shock flow.

The center of the V605 Aql nebula is optically thick (Pollacco et al. 1992) so the back side flow can not be seen. Again this suggests an origin for this gas at the central disk. Assuming the $3.8 \mathrm{kpc}$ distance, the visible nebula size, with a radius of $\sim 3000 \mathrm{AU}$, is in rough agreement with an outflow velocity of $\sim 140 \mathrm{~km} \mathrm{~s}^{-1}$ for 80 years if the visible nebula probes the first mass ejected. The optical nebular lines in V605 Aql have widths and velocities that are in general agreement with similar outflow velocities seen in A30 and A78 (Harrington et al. 1995; Yadoumaru \& Tamura 1994), suggesting that the origin of the nebula in a [WR] wind and subsequent deceleration of the wind in a disk is a common process to all these objects.

Comparison of archival HST images of V605 Aql (Sect. 3.1) with the images presented in this paper would indicate expansion rates of $\gtrsim 1000 \mathrm{~km} \mathrm{~s}^{-1}$. Spectroscopy of the nebula does not support the presence of velocities this large except near the [WR] object. Furthermore the size of the shell is far too small for expansion at this rate since the 1920s. The archival observations 
are of inferior quality to the new observations so it is likely that the increase in size and hence the implied expansion velocity is largely due to increased depth in the newer images. We note that the ground based images of Guerrero \& Manchado (1996) have a larger diameter than the current images. All three sets of images do have morphological similarity.

\subsection{Origin of the disk}

Four general processes have been suggested for the formation of bipolar nebulae (Soker \& Rappaport 2000). Three of these processes involve binary systems and one involves stellar rotation. Discriminating between the binary and rotation processes can be non-trivial. Detailed discussions relating these process to the origin of PNe can be found in, e.g., Soker (1997) and Moe \& De Marco (2006).

Soker (1997) lists the V605 Aql hydrogen-rich PNe A58 as having a high confidence of originating from common envelope evolution with a substellar companion. If this is the case then the AGB star had a substellar companion that underwent a common envelope stage. De Marco \& Soker (2002) speculate that [WR] central stars of planetary nebulae are of binary origin. The companion or perhaps a more outlying substellar companion would then have to survive the common envelope stage to be a companion in the final flash stage. This companion could then engage in a second common envelope stage. The mass loss of the V605 Aql system is ongoing through a disk. If driven by a binary the surviving companion to the [WR] star must be close; the companion must be interior to the inner disk radius of $\sim 3 \mathrm{AU}$. Binary systems are known to restrict mass loss to a thick disk in the post-binary HR 4049 systems (Hinkle et al. 2007). However, there is no independent, for instance radial velocity, evidence that V605 Aql or any other final flash object is a binary.

The alternate process for symmetric nebula is rotation of a single star undergoing mass loss (Garcia-Segura et al. 1999; Reimers et al.2000) An interesting feature of V605 Aql, A30, and A78 is that the old, hydrogen rich PNe have only slightly oblate shells (Sect. 3; Jacoby 1979). The major axis of A58 is aligned with the V605 Aql disk axis (Fig. 1). If the mass loss in a disk is due to stellar rotation the implication is that rotation has greatly increased from the AGB to final flash stages.

\section{Conclusions}

The near-infrared colors of V605 Aql require hot, 1500 K, dust. Previous work by Pollacco et al. (1992), Clayton et al. (2006), and others shows that the central star in V605 Aql is surrounded by an optically thick disk inclined to the line of sight and obscuring the central star. Spectroscopy of V605 Aql reveals the presence of He I $10830 \AA$, a spectral line identified with a shock as high velocity gas interacts with a dust layer (Clayton 2003). A high velocity, $2500 \mathrm{~km} \mathrm{~s}^{-1}$, wind is known to flow off the hot [WR] remnant (Clayton et al. 2006).

As in FG Sge (Gehrz et al.2005), we suggest that the current [WR] wind has been flowing from V605 Aql since dust condensed, i.e. for the last 80 years. The He I $10830 \AA$ occurs near a shock where this wind encounters the inner edge of the circumstellar disk. The hot dust at this shock zone is the origin of the $1500 \mathrm{~K}$ emission. The $1500 \mathrm{~K}$ region is visible because the disk surrounding the star is inclined to the line of sight. The dust then flows into an optically thick disk. The dust is at $350 \mathrm{~K}$ when the disk becomes optically thin in the infrared. Simple arguments suggest an inner boundary at $\sim 3 \mathrm{AU}$ from the central star and a outer boundary at $\sim 60 \mathrm{AU}$. The velocity of post-shock gas is $\sim 140 \mathrm{~km} \mathrm{~s}^{-1}$, which accounts for the velocity of the gas in the optical nebular spectrum. The shadow of the disk is clearly visible in the high spatial resolution HST images.

The implication is that the dust formation event in final flash stars marks the transition from an optically thick pseudophotosphere to the emergence of a hot white dwarf surrounded by a dust disk of its own making. A [WR] stellar wind starts at this time but a single ejection of a shell of material did not occur for V605 Aql. The mass lost from V605 Aql as a result of 80 years of [WR] wind is $\sim 1 \times 10^{-5} M_{\odot}$. The theoretically expected mass loss of $\sim 10^{-4}-10^{-3} M_{\odot}$ will take $\sim 10^{3}-10^{4} \mathrm{yrs}$. The circumstellar V605 Aql nebula is the expanding bubble of material blow out by the decelerated [WR] wind. The $140 \mathrm{~km} \mathrm{~s}^{-1}$ post-shock wind has had time to blow a bubble $\sim 3000 \mathrm{AU}$ in radius in 80 years and has overtaken bubbles of dust lost at lower velocity in the RCB stage of the final flash.

While the much older final flash objects A30 and A78 show extended circumstellar shells with intense wind-interaction, these objects also have dual dust temperature components at $\sim 140 \mathrm{~K}$ and $\sim 1000 \mathrm{~K}$ (Jacoby 1979; Dinerstein et al. 1984) which are diagnostic of a disk. We believe the [WR] disk phase of the final flash is still going on in the old final flash objects A30 and A78. We note that there are striking similarities between all the final flash objects (Table 1). We suggest that a disk mass loss stage is occurring in all final flash objects that have evolved past the pseudo-photosphere stage.

Acknowledgements. Based in part on observations made with the NASA/ESA Hubble Space Telescope, obtained at the Space Telescope Science Institute, which is operated by the Association of Universities for Research in Astronomy, Inc., under NASA contract NAS 5-26555. Based in part on observations obtained at the Gemini Observatory, which is operated by the Association of Universities for Research in Astronomy, Inc., under a cooperative agreement with the NSF on behalf of the Gemini partnership: the National Science Foundation (United States), the Particle Physics and Astronomy Research Council (United Kingdom), the National Research Council (Canada), CONICYT (Chile), the Australian Research Council (Australia), CNPq (Brazil), and CONICRT (Argentina) with the Adaptive Optics System Hokupa'a/QUIRC, developed and operated by the University of Hawaii Adaptive Optics Group, with support from the National Science Foundation. Based in part on observations collected at the European Southern Observatory, Chile.

Thomas Lebzelter was supported by the Austrian Science Fund Project P18171. Josef Hron and Karin Andre were supported by the Austrian Ministry for Education, Science and Culture. We would like to thank M. Sterzik (ESO) for his assistance with the TIMMI2 observations. Software created by Martin Sperl was essential in the reduction of the TIMMI 2 observations. We would also like to thank Olivier Guyon, Dan Potter, and Francois Rigaut for their assistance with the Gemini North observations. This research made use of the SIMBAD database operated by CDS in Strasbourg, France and NASA's Astrophysics Data System Bibliographic Services. We thank Geoff Clayton for insightful comments, especially concerning the RCB stage, that highly flavored the discussion.

\section{References}

Acker, A., \& Neiner, C. 2003, A\&A, 403, 659

Althaus, L. G., Serenelli, A. M., Panei, J. A., et al. 2005, A\&A, 435, 631

Asplund, M., Gustafsson, B., Lambert, D. L., \& Kameswara Rao, N. 1997, A\&A, 321, L17

Asplund, M., Lambert, D. L., Kipper, T., Pollacco, D., \& Shetrone, M. D. 1999, A\&A, 343, 507

Balick, B. 1989, in Planetary Nebulae, ed. S. Torres-Peimbert (Kluwer: Dordrecht), IAU Symp., 131, 83

Blöcker, T. 2001, Ap\&SS, 275, 1

Blöcker, T., \& Schönberner, D. 1997, A\&A, 324, 991

Bond, H. E., \& Pollacco, D. L. 2002, Ap\&SS, 279, 31

Borkowski, K. J., Harrington, J. P., Tsvetanov, Z. I., \& Clegg, R. E. 1993, ApJ, 415, L47

Borkowski, K. J., Harrington, J. P., Blair, W. P., \& Bregman, J. D. 1994, ApJ, 435, 722

Borkowski, K. J., Harrington, J. P., \& Tsvetanov, Z. I. 1995, ApJ, 449, L143 
Cahn, J. H., \& Kaler, J. B. 1971, ApJS, 22, 319

Clayton, G. C., \& De Marco, O. 1997, AJ, 114, 2679

Clayton, G. C., Geballe, T. R., \& Bianchi, L. 2003, ApJ, 595, 412

Clayton, G. C., Kerber, F., Pirzkal, N., et al. 2006, ApJ, 646, L69

Clayton, G. C., Geballe, T. R., Herwig, F., Fryer, C., \& Asplund, M. 2007, ApJ, 662,1220

Clegg, R. E. S., Devaney, M. N., Doel, A. P., et al. 1993, in Planetary Nebulae, ed. R. Weinberger, \& A. Acker (Dordrecht: Kluwer), IAU Symp., 155, 388

Cohen, M., Van Winckel, H., Bond, H. E., \& Gull, T. R. 2004, AJ, 127, 2362

De Marco, O., \& Soker, N. 2002, PASP, 114, 602

Dinerstein, H. L., \& Lester, D. F. 1984, ApJ, 281, 702

Dominik, C., Dullemond, C. P., Cami, J., \& van Winckel, H. 2003, A\&A, 397, 595

Draine, B. T. 1981, ApJ, 245, 880

Duerbeck, H. W., \& Benetti, S. 1996, ApJ, 468, 111

Evans, A., van Loon, J. Th., Zijlstra, A. A., et al. 2002, MNRAS, 332, L35

Eyres, S. P. S., Smalley, B., Geballe, T. R., et al. 1999, MNRAS, 307, L11

Eyres, S. P. S., Geballe, T. R., Yyne, V. H., et al. 2004, MNRAS, 350, L9

García-Lario, P., Manchado, A., Pych, W., \& Pattasch, S. R. 1997, A\&AS, 126, 479

Garcia-Segura, G., Langer, N., Rozyczka, M., \& Franco, J. 1999, ApJ, 517, 767

Gehrz, R. D. 1999, Phys. Rep., 311, 405

Gehrz, R. D., Woodward, C. E., Temin, T., Lyke, J. E., \& Mason, C. G. 2005, ApJ, 623, 1105

Gonzalez, G., Lambert, D. L., Wallerstein, G., et al. 1998, ApJS, 114, 133

Górny, S. K. 2001, Ap\&SS, 275, 67

Górny, S. K., \& Tylenda, R. 2000, A\&A, 362, 1008

Górny, S. K., Stasinska, G., Szczerba, R., \& Tylenda, R. 2001, A\&A, 377, 1007

Graves, J. E., Northcott, M. J., Roddier, F. J., Roddier, C. A., \& Close, L. M. 1998, Proc. Spie, 3353, 34

Graves, J. E., Northcott, M. J., Roddier, F. J., et al. 2000, Proc. Spie, 4007, 26

Guerrero, M. A., \& Manchado, A. 1996, ApJ, 472, 711

Harrington, J. P., \& Feibelman, W. A. 1984, ApJ, 277, 716

Harrington, J. P., Borkowski, K. J., \& Tsvetanov, Z. 1995, ApJ, 439, 264

Hartigan, P., Raymond, J., \& Hartmann, L. 1987, ApJ, 316, 323

Herwig, F. 2005, ARA\&A, 43, 435

Herwig, F., Blöcker, T., Langer, N., \& Driebe, T. 1999, A\&A, 349, L5

Hinkle, K. H., \& Joyce, R. R. 2002, Ap\&SS, 279, 51

Hinkle, K. H., Joyce, R. R., \& Smith, V. V. 1995, AJ, 109, 808

Hinkle, K. H., Joyce, R. R., \& Hedden, A. 2001, A\&A, 367, 250

Hinkle, K. H., Brittain, S. D., \& Lambert, D. L. 2007, ApJ, 664, 501

Iben, I. Jr. 1984, ApJ, 277, 333

Iben, I. Jr., Kaler, J. B., Truran, J. W., \& Renzini, A. 1983, ApJ, 264, 605 Jacoby, G. 1979, PASP, 91, 754

Jacoby, G. H., \& Ford, H. C. 1983, ApJ, 266, 298

Jeffrey, C. S., \& Schönberner, D. 2006, A\&A459, 885

Joyce, R. R., Fowler, A. M., \& Heim, G. B. 1994, Proc. Spie, 2198, 725

Kaler, J. B., Feibelman, W. A., \& Henrichs, H. F. 1988, ApJ, 324, 528

Käufl, H. U., Koller, J., \& Kerber, F. 2003, A\&A, 406, 981
Kerber, F., Pirzkal, N., De Marco, O., et al. 2002, ApJ, 581, L39

Kimeswenger, S. 2003, Rev. Mex. Astron. Astrofis. Ser. Conf., 15, 75

Kimeswenger, S., Kerber, F., \& Weinberger, R. 1998, MNRAS, 296, 614

Kimeswenger, S., Koller, J., \& Schmeja, S. 2000, A\&A, 360, 699

Koller, J., \& Kimeswenger, S. 2001, ApJ, 559, 419

Kurucz, R. L. 1979, ApJS, 40,

Kwok, S. 1982, ApJ, 258, 280

Lambert, D. L., Rao, N. K., Pandey, G., \& Ivans, I. I. 2001, ApJ, 555, 925

Lawlor, T. M., \& MacDonald, J. 2003, ApJ, 583, 913

Lechner, M. F. M., \& Kimeswenger, S. 2004, A\&A, 426, 145

Leuenhagen, U., Koesterke, L., \& Hamann, W.-R. 1993, AcAst, 43, 329

Maciel, J. W. 1984, A\&AS, 55, 253

Meaburn, J., López, J. A., Bryce, M., \& Redman, M. P. 1998, A\&A, 334, 670

Miller Bertolami, M. M., Althaus, L. G., Serenelli, A. M., \& Panei, J. A. 2006, A\&A449, 313

Misselt, K. A., Gordon, K. D., Clayton, G. C., \& Wolff, M. J. 2001, ApJ, 551, 277

Moe, M., \& De Marco, O. 2006, ApJ, 650, 916

Pavlenko, Ya. V., Geballe, T. R., Evans, A., et al. 2004, A\&A, 417, L39

Phillips, J. P., \& Ramos-Larios, G. 2006, MNRAS, 368, 1773

Pollacco, D. L., Lawson, W. A., Clegg, R. E. S., \& Hill, P. W. 1992, MNRAS, $257,33 p$

Reimann, H.-G., Linz, H., Wagner, R., et al. 2000, in Optical and IR Telescope Instrumentation and Detectors, ed. M. Iye, \& A. F. Moorwood, Proc. Spie, 4008, 1132

Reimers, Ch., Dorfi, E. A., \& Höfner, S. 2000, A\&A, 354, 573

Relke, H., Sperl, M., Hron, J., et al. 2000, in Advanced Telescope and Instrumentation Control Software, ed. H. Lewis, Proc. Spie, 4009, 440

Seitter, W. C. 1987, Messenger, 50, 14

Sharp, C. M., \& Wasserburg, G. J. 1995, Geochimica et Cosmochimica Acta, 59, 1633

Soker, N. 1997, ApJS, 112, 487

Soker, N., \& Rappaport, S. 2000, ApJ, 538, 241

Tyne, V. H., Eyres, S. P. S., Geballe, T. R., et al. 2000, MNRAS, 315, 595

Tyne, V. H., Evans, A., Geballe, T. R., et al. 2002, MNRAS, 334, 875

van der Hucht, K. A., Conti, P. S., Lundstrom, I., \& Stenholm, B. 1981, Space

Sci. Rev., 28, 227

van der Veen, W. E. C. J., Habing, H. J., \& Geballe, T. R. 1989, A\&A, 226, 108

van Hoof, P. A. M., Bryce, M., Evans, A., et al. 2006, in Planetary Nebulae in

Our Galaxy, ed. M. J. Barlow, \& R. H. Méndez, 119

van Hoof, P. A. M., Hajduk, M., Zijlstra, A. A., et al. 2007, A\&A, 471, L9

Werner, K., \& Herwig, F. 2006, PASP, 118, 183

Werner, K., \& Koesterke, L. 1992, in The Atmospheres of Early-Type Stars, ed.

U. Heber, \& C. S. Jeffery (Berlin: Springer-Verlag), 288

Whitelock, P. A. 1985, MNRAS, 213, 59

Wolf, M. 1920, Astron. Nachr., 211, 119

Yadoumaru, Y., \& Tamura, S. 1994, PASP, 106, 165

Zijlstra, A. A. 2002, Ap\&SS, 279, 171 